\title{
Kinetic energy sum spectra in nonmesonic weak decay of hypernuclei
}

\author{
Cesar Barbero, ${ }^{1,4}$ Alfredo P. Galeão, ${ }^{2}$ Mahir S. Hussein, ${ }^{3,5}$ and Francisco Krmpoticic $3,4,6$ \\ ${ }^{1}$ Facultad de Ciencias Exactas, Departamento de Física, Universidad Nacional de La Plata, 1900 La Plata, Argentina \\ ${ }^{2}$ Instituto de Física Teórica, Universidade Estadual Paulista, Rua Pamplona 145, 01405-900 São Paulo, SP, Brazil \\ ${ }^{3}$ Departamento de Física Matemática, Instituto de Física da Universidade de São Paulo, Caixa Postal 66318, 05315-970 São Paulo, SP, Brazil \\ ${ }^{4}$ Instituto de Física La Plata, CONICET, 1900 La Plata, Argentina \\ ${ }^{5}$ Max-Planck-Institut für Physik komplexer Systeme, Nöthnitzer Straße 38, D-01187 Dresden, Germany \\ ${ }^{6}$ Facultad de Ciencias Astronómicas y Geofísicas, Universidad Nacional de La Plata, 1900 La Plata, Argentina \\ (Received 21 February 2008; revised manuscript received 18 July 2008; published 16 October 2008; \\ publisher error corrected 29 October 2008)
}

\begin{abstract}
We evaluate the coincidence spectra in the nonmesonic weak decay (NMWD) $\Lambda N \rightarrow n N$ of $\Lambda$ hypernuclei ${ }_{\Lambda}^{4} \mathrm{He},{ }_{\Lambda}^{5} \mathrm{He},{ }_{\Lambda}^{12} \mathrm{C},{ }_{\Lambda}^{16} \mathrm{O}$, and ${ }_{\Lambda}^{28} \mathrm{Si}$, as a function of the sum of kinetic energies $E_{n N}=E_{n}+E_{N}$ for $N=n, p$. The strangeness-changing transition potential is described by the one-meson-exchange model, with commonly used parametrization. Two versions of the independent-particle shell model (IPSM) are employed to account for the nuclear structure of the final residual nuclei. They are as follows: (a) IPSM-a, where no correlation, except for the Pauli principle, is taken into account and (b) IPSM-b, where the highly excited hole states are considered to be quasistationary and are described by Breit-Wigner distributions, whose widths are estimated from the experimental data. All $n p$ and $n n$ spectra exhibit a series of peaks in the energy interval $110 \mathrm{MeV}<E_{n N}<$ $170 \mathrm{MeV}$, one for each occupied shell-model state. Within the IPSM-a, and because of the recoil effect, each peak covers an energy interval proportional to $A^{-1}$, going from $\cong 4 \mathrm{MeV}$ for ${ }_{\Lambda}^{28} \mathrm{Si}$ to $\cong 40 \mathrm{MeV}$ for ${ }_{\Lambda}^{4} \mathrm{He}$. Such a description could be pretty fair for the light ${ }_{\Lambda}^{4} \mathrm{He}$ and ${ }_{\Lambda}^{5} \mathrm{He}$ hypernuclei. For the remaining, heavier, hypernuclei it is very important, however, to consider as well the spreading in strength of the deep-hole states and bring into play the IPSM-b approach. Notwithstanding the nuclear model that is employed the results depend only very weakly on the details of the dynamics involved in the decay process proper. We propose that the IPSM is the appropriate lowest-order approximation for the theoretical calculations of the of kinetic energy sum spectra in the NMWD. It is in comparison to this picture that one should appraise the effects of the final-state interactions and of the two-nucleon-induced decay mode.
\end{abstract}

DOI: 10.1103/PhysRevC.78.044312

PACS number(s): 21.80.+a, 13.75.Ev, 21.60.-n, 21.10.Pc

\section{INTRODUCTION}

Because of the difficulty in detecting neutrons, until a few years ago only the high-energy proton spectra had been measured in the two-body $\Lambda N \rightarrow n N$ nonmesonic weak decay (NMWD) of $\Lambda$ hypernuclei. The proton-induced transition rates $\Gamma_{p} \equiv \Gamma(\Lambda p \rightarrow n p)$ in ${ }_{\Lambda}^{5} \mathrm{He},{ }_{\Lambda}^{11} \mathrm{Li}$, and ${ }_{\Lambda}^{12} \mathrm{C}$ have been determined in this way [1-3]. The corresponding neutroninduced transition rates $\Gamma_{n} \equiv \Gamma(\Lambda n \rightarrow n n)$ were estimated through the comparison of the measured proton spectrum with that of the intranuclear cascade (INC) calculation where the $\Gamma_{n} / \Gamma_{p}$ ratio is treated as free parameter. This procedure signalized very large experimental $n / p$ ratios $(\cong 0.9-2.0$ ) $[1-3]$, in comparison with theoretical estimates $(\cong 0.3-0.5)$ obtained within the one-meson-exchange potential (OMEP) [4-11]. (See, for instance, Table 3 in Ref. [7].) In spite of large uncertainties involved in the indirect evaluation of neutrons, this discrepancy between data and theory was considered to be a serious puzzle in the NMWD.

Yet, quite recently, the above scenario has drastically changed due to the very significant advances in our knowledge (mainly experimental) on both neutron and proton spectra. These advances are as follows:

(i) In the experiment E369 were measured high-quality neutron spectra in the decays of ${ }_{\Lambda}^{12} \mathrm{C}$ and ${ }_{\Lambda}^{89} \mathrm{Y}$, which made possible to compare them directly with the corresponding proton spectra, yielding the result $\Gamma_{n} / \Gamma_{p}=$ $(0.45-0.51) \pm 0.15$ [12].

(ii) Okada et al. [13] have simultaneously measured the energy spectra of neutrons and protons in ${ }_{\Lambda}^{5} \mathrm{He}$ and ${ }_{\Lambda}^{12} \mathrm{C}$ at a high energy threshold $(60 \mathrm{MeV})$, from where it was inferred that for both hypernuclei $\Gamma_{n} / \Gamma_{p} \cong 0.5$.

(iii) Garbarino, Parrẽno, and Ramos [14-16] have called attention on the fact that "correlation observables permit a cleaner extraction of $\Gamma_{n} / \Gamma_{p}$ from data than single-nucleon observables," which has stimulated several experimental searches [17-20]. They have also done a theoretical evaluation of the pair distributions for: (a) the sum of the $n N$ kinetic energies $E=E_{n}+$ $E_{N}, S_{n N}(E)$ and (b) the opening angle $\theta, S_{n N}(\cos \theta)$, in the decays of ${ }_{\Lambda}^{5} \mathrm{He}$ and ${ }_{\Lambda}^{12} \mathrm{C}$. The number of detected $n N$ pairs $N_{n N}$ is proportional to $\Gamma_{N}=\int S_{n N}(E) d E=$ $\int S_{n N}(\cos \theta) d \cos \theta$ and therefore $N_{n n} / N_{n p} \cong \Gamma_{n} / \Gamma_{p}$ (see below). The primary weak decay was described by the OMEP dynamics within the framework of a shell model, whereas an INC code was used to take into account the strong final-state interactions (FSI) involving the primary nucleons and those in the residual nucleus, including the possibility of emission of secondary particles. They conclude that the datum 
$N_{n n} / N_{n p}=0.44 \pm 0.11$ obtained in KEK-E462 [17] for ${ }_{\Lambda}^{5} \mathrm{He}$ is compatible with an $n / p$ ratio of $0.39 \pm 0.11$ for this hypernucleus if the two-nucleon induced mode $\Lambda N N \rightarrow n N N$ is neglected, or an even lower value if it is included. Similarly, together with Bauer [21], they found $\Gamma_{n} / \Gamma_{p}\left({ }_{\Lambda}^{12} \mathrm{C}\right)=0.46 \pm 0.09$ when the twonucleon induced mode is neglected and a slightly lower value when it is included. The shell-model used in Refs. [14-16] is substituted in the last work by a nuclear matter formalism extended to finite nuclei via the local density approximation, whereas the Monte Carlo INC model is retained to account for the FSI.

(iv) Quite recently Bauer [22] has given a step forward with his nuclear matter formalism, describing microscopically both the weak decay mechanism and the FSI, confirming in this way that the latter lead to somewhat lower value for the ratio $\Gamma_{n} / \Gamma_{p}$ in ${ }_{\Lambda}^{12} \mathrm{C}$.

(v) Several coincidence emission measurements of the above mentioned spectra have been performed [17-20, $23,24]$, from which were extracted the new experimental results, $\Gamma_{n} / \Gamma_{p}\left({ }_{\Lambda}^{5} \mathrm{He}\right)=0.45 \pm 0.11 \pm 0.03$ and $\Gamma_{n} / \Gamma_{p}\left({ }_{\Lambda}^{12} \mathrm{C}\right)=0.40 \pm 0.09$, that point toward the solution of the longstanding $\Gamma_{n} / \Gamma_{p}$ puzzle. $^{1}$

Reasoning within the two-body kinematics for the onenucleon induced NMWD, it is expected that $S_{n N}(E)$ should exhibit a narrow peak at the two-particle energy $E$ close to the decay $Q$ value, whereas $S_{n N}(\cos \theta)$ should be restrained within the back-to-back angle $\theta \cong \pi$. Thus, all experimentally observed deviations from such spectral shapes are very frequently attributed to the FSI and/or to the two-nucleon induced processes [17-20,23,24]. ${ }^{2}$ However, this is no longer the case when the recoil of the residual nucleus is taken into account, which makes the kinematics to be of a three-body type. Moreover, when the shell-model structure is also taken into account the energy spectra will have a bump at each single-particle state, the width of which will depend on the magnitude of both the recoil and the spreading in strength of the hole states in the inner shells. Although the detailed structure and fragmentation of hole states are still not well known, the exclusive knockout reactions provide a wealth of information on the structure of single-nucleon states of nuclei. Excitation energies and widths of proton-hole states were systematically measured with quasifree $(p, 2 p)$ and $\left(e, e^{\prime} p\right)$ reactions, which revealed the existence of inner orbital shells in nuclei [26-34].

The aim of the present work is to discuss quantitatively the interplay between the recoil effect and the nuclear shell structure in the kinetic energy sum spectra of NMWD. The article is organized as follows. In Sec. II we discuss the calculation of these spectra within the independent-particle

\footnotetext{
${ }^{1}$ We note that the relationship $\Gamma_{n} / \Gamma_{p}\left({ }_{\Lambda}^{5} \mathrm{He}\right) \sim \Gamma_{n} / \Gamma_{p}\left({ }_{\Lambda}^{12} \mathrm{C}\right)$ could have a very simple explanation, similar to that given in Ref. [25] for the asymmetry parameter: $a_{\Lambda}\left({ }_{\Lambda}^{5} \mathrm{He}\right) \sim a_{\Lambda}\left({ }_{\Lambda}^{12} \mathrm{C}\right)$.

${ }^{2}$ In Refs. $[14,15]$ it is said that the $n p$ energy spectra of the three-body proton-induced decay ${ }_{\Lambda}^{5} \mathrm{He} \rightarrow{ }^{3} \mathrm{H}+n+p$ should exhibit a narrow peak close to its $Q$ value of $153 \mathrm{MeV}$, which is only valid when the recoil effect is neglected.
}

shell-model (IPSM). In Sec. III we exhibit the numerical results for ${ }_{\Lambda}^{4} \mathrm{He},{ }_{\Lambda}^{5} \mathrm{He},{ }_{\Lambda}^{12} \mathrm{C},{ }_{\Lambda}^{16} \mathrm{O}$, and ${ }_{\Lambda}^{28} \mathrm{Si}$ hypernuclei. In Sec. IV we discuss these results and their connection with the experimental data. Finally, in Sec. V, we present several concluding remarks.

\section{KINETIC ENERGY SUM SPECTRUM}

For the purpose of completeness and clarity, we will first redo the calculation of the decay rate $\Gamma_{N}$ using Fermi's golden rule. The novelties here, in comparison with our previous works Refs. [6-8], are the recoil effect and the spreading of the single-particle configurations. As will be shown in Sec. IV, their role is of minor importance in the evaluation of the integrated transition rates $\Gamma_{N}$, as well as on the ratio $\Gamma_{n} / \Gamma_{p}$, but they are crucial for a correct description of the energy distribution of the transition strength. In fact, a single-particle state $\left|j_{N}\right\rangle$ that is deeply bound in the hypernucleus, after the NMWD can become a highly excited hole-state $\left|j_{N}^{-1}\right\rangle$ in the continuum of the residual nucleus. There it suddenly mixes up with more complicated configurations $(2 \mathrm{~h} 1 \mathrm{p}$, $3 \mathrm{~h} 2 \mathrm{p}, \ldots$ excitations, collective states, etc.) spreading its strength in a relatively wide energy interval [35]. ${ }^{3}$ This happens, for instance, with the $1 s_{1 / 2}$ orbital in ${ }_{\Lambda}^{12} \mathrm{C}$, that is separated from the $1 p_{3 / 2}$ state by approximately $23 \mathrm{MeV}$, which is enough to break the 10-particle system, where the energy of the last excited state amounts to $\sim 16.5 \mathrm{MeV}$.

The NMWD rate of a hypernucleus (in its ground state with spin $J_{I}$ and energy $E_{J_{I}}$ ) to residual nuclei (in the several allowed states with spins $J_{F}$ and energies $E_{\alpha_{N} J_{F}}$ ) and two free nucleons $n N$ (with total spin $S$ and total kinetic energy $\left.E_{n N}=E_{n}+E_{N}\right)$, reads

$$
\begin{aligned}
\Gamma_{N}= & 2 \pi \sum_{S M_{S} \alpha_{N} J_{F} M_{F}} \int\left|\left\langle\mathbf{p}_{n} \mathbf{p}_{N} S M_{S} ; \alpha_{N} J_{F} M_{F}|V| J_{I} M_{I}\right\rangle\right|^{2} \\
& \times \delta\left(\Delta_{\alpha_{N} J_{F}}-E_{r}-E_{n N}\right) \frac{d \mathbf{p}_{n}}{(2 \pi)^{3}} \frac{d \mathbf{p}_{N}}{(2 \pi)^{3}}
\end{aligned}
$$

Here, $V$ is the hypernuclear nonmesonic weak transition potential, and the wave functions for the kets $\left|\mathbf{p}_{n} \mathbf{p}_{N} S M_{S} ; \alpha_{N} J_{F} M_{F}\right\rangle$ and $\left|J_{I} M_{I}\right\rangle$ are assumed to be antisymmetrized and normalized. The label $\alpha_{N}$ stands for different final states with the same spin $J_{F}, E_{r}$ is the recoil energy of the residual nucleus, and

$$
\Delta_{\alpha_{N} J_{F}}=\Delta+E_{J_{I}}-E_{\alpha_{N} J_{F}}
$$

with

$$
\Delta=M_{\Lambda}-M=176 \mathrm{MeV},
$$

is the liberated energy. The two emitted nucleons are described by plane waves, and initial and final short-range correlations are included phenomenologically at a simple Jastrow-like level.

\footnotetext{
${ }^{3}$ One should keep in mind that the mean life a $\Lambda$ hyperon is $\tau_{\Lambda}=$ $2.63 \times 10^{-10} \mathrm{~s}$, whereas the strong interaction times are of the order of $10^{-21} \mathrm{~s}$.
} 
It is convenient to perform a transformation to the relative and center-of-mass momenta $\left[\mathbf{p}=\frac{1}{2}\left(\mathbf{p}_{n}-\mathbf{p}_{N}\right), \mathbf{P}=\mathbf{p}_{n}+\right.$ $\mathbf{p}_{N}$ ], coordinates $\left[\mathbf{r}=\mathbf{r}_{n}-\mathbf{r}_{N}, \mathbf{R}=\frac{1}{2}\left(\mathbf{r}_{n}+\mathbf{r}_{N}\right)\right]$ and orbital angular momenta $\mathbf{I}$ and $\mathbf{L}$, and to express the energy conservation as

$$
E_{n N}+E_{r}-\Delta_{\alpha_{N} J_{F}}=\epsilon_{p}+\epsilon_{P}-\Delta_{\alpha_{N} J_{F}}=0,
$$

where

$$
\begin{aligned}
\epsilon_{p} & =\frac{p^{2}}{M}, \quad E_{r}=\frac{P^{2}}{2 M(A-2)}, \\
\epsilon_{P} & =\frac{P^{2}}{4 M} \frac{A}{A-2}=\frac{A}{2} E_{r},
\end{aligned}
$$

are, respectively, the energies of the relative motion of the outgoing pair, of the recoil, and of the total center-of-mass motion (including the recoil). Following step by step the analytical developments done in Ref. [6], the transition rate can be can expressed as

$$
\Gamma_{N}=\int_{0}^{\Delta} \frac{d \Gamma_{N}}{d \epsilon_{P}} d \epsilon_{P}
$$

where we have defined (see Eqs. (2.13) and (2.14) in Ref. [6])

$$
\begin{aligned}
& \frac{d \Gamma_{N}}{d \epsilon_{P}} \\
& =\frac{16 M^{3}}{\pi}\left(\frac{A-2}{A}\right)^{3 / 2} \hat{J}_{I}^{-2} \sum_{S \lambda l L T J \alpha_{N} J_{F}} \sqrt{\epsilon_{P}\left(\Delta_{\alpha_{N} J_{F}}-\epsilon_{P}\right)} \\
& \quad \times\left|\sum_{j_{N}} \mathcal{M}\left(p P l L \lambda S J T ; j_{N}\right)\left(J_{I}\left\|\left(a_{j_{N}}^{\dagger} a_{j_{\Lambda}}^{\dagger}\right)_{J}\right\| \alpha_{N} J_{F}\right\rangle\right|^{2},
\end{aligned}
$$

it being understood that the square root is to be replaced by zero whenever its argument is negative. The angular momentum couplings $\mathbf{I}+\mathbf{L}=\lambda$ and $\lambda+\mathbf{S}=\mathbf{J}$ have been carried out, $\hat{J} \equiv \sqrt{2 J+1}$, and $A=Z+N+1$ is the total number of baryons.

It is self-evident that for $A \rightarrow \infty$ one obtains the same result as in Refs. [6-8]. It is also worth noting that the overall outcome of the recoil on $\Gamma_{N}$ is very small, mostly because the effect of the factor $[(A-2) / A]^{3 / 2}$ in Eq. (6) is, to a great extent, canceled by the effect of the factor $[A /(A-2)]^{3 / 2}$ originating from $\sqrt{\epsilon_{P}\left(\Delta_{\alpha_{N} J_{F}}-\epsilon_{P}\right)} d \epsilon_{P}$. This is the reason why we have not included the recoil previously.

The spectrum of $\Gamma_{N}$ as a function of $E_{n N}$ is now easily obtained from Eq. (6) by means of the relation

$$
E_{n N}=\Delta_{\alpha_{N} J_{F}}-\frac{2}{A} \epsilon_{P}
$$

as follows from Eqs. (3) and (4). Calling $E \equiv E_{n N}$, one gets

$$
\Gamma_{N}=\int_{0}^{\Delta} S_{n N}(E) d E
$$

with

$$
\begin{aligned}
S_{n N}(E)= & \frac{4 M^{3}}{\pi} \sqrt{A(A-2)^{3}} \hat{J}_{I}^{-2} \\
& \times \sum_{S \lambda l L T J \alpha_{N} J_{F}} \sqrt{\left(\Delta_{\alpha_{N} J_{F}}-E\right)\left(E-\Delta_{\alpha_{N} J_{F}}^{\prime}\right)} \\
\times & \left|\sum_{j_{N}} \mathcal{M}\left(p P l L \lambda S J T ; j_{N}\right)\left\langle J_{I}\left\|\left(a_{j_{N}}^{\dagger} a_{j_{\Lambda}}^{\dagger}\right)_{J}\right\| \alpha_{N} J_{F}\right)\right|^{2},
\end{aligned}
$$

where

$$
\begin{gathered}
p=\sqrt{\frac{M A}{2}\left(E-\Delta_{\alpha_{N} J_{F}}^{\prime}\right)}, \\
P=\sqrt{2 M(A-2)\left(\Delta_{\alpha_{N} J_{F}}-E\right)}, \\
\Delta_{\alpha_{N} J_{F}}^{\prime}=\Delta_{\alpha_{N} J_{F}} \frac{A-2}{A},
\end{gathered}
$$

and the condition

$$
\Delta_{\alpha_{N} J_{F}}^{\prime} \leqslant E \leqslant \Delta_{\alpha_{N} J_{F}}
$$

has to be fulfilled for each contribution.

As previously [6-8], it will be assumed that the hyperon in the state $j_{\Lambda}$, with single-particle energy $\epsilon_{j_{\Lambda}}$, is weakly coupled to the $A-1$ core, with spin $J_{C}$ and energy $E_{C}=$ $E_{J_{I}}-\epsilon_{j_{\Lambda}}$. Then the initial state is $\left|J_{I}\right\rangle \equiv\left|\left(J_{C} j_{\Lambda}\right) J_{I}\right\rangle$, and the spectroscopic amplitude $\left\langle J_{I}||\left(a_{j_{N}}^{\dagger} a_{j_{\Lambda}}^{\dagger}\right)_{J}|| \alpha_{N} J_{F}\right\rangle$ can be rewritten as

$$
\begin{aligned}
\left\langle J_{I}\left\|\left(a_{j_{N}}^{\dagger} a_{j_{\Lambda}}^{\dagger}\right)_{J}\right\| \alpha_{N} J_{F}\right\rangle= & (-)^{J_{F}+J+J_{I}} \hat{J} \hat{J}_{I}\left\{\begin{array}{ccc}
J_{C} & J_{I} & j_{\Lambda} \\
J & j_{N} & J_{F}
\end{array}\right\} \\
& \times\left\langle J_{C}\left\|a_{j_{N}}^{\dagger}\right\| \alpha_{N} J_{F}\right\rangle .
\end{aligned}
$$

The following two approaches for the final states $\left|\alpha_{N} J_{F}\right\rangle$ will be examined within the IPSM.

\section{A. IPSM-a}

Here, we completely ignore the residual interaction and, consequently, the only states $\left|\alpha_{N} J_{F}\right\rangle$ giving a nonzero result in Eq. (13) and therefore contributing to Eq. (9) are those obtained by the weak coupling, and properly antisymmetrizing, of the one-hole (1h) states $\left|j_{N}^{-1}\right\rangle$ to the core ground state $\left|J_{C}\right\rangle$. That is, recalling that we completely ignore the residual interaction in this approximation,

$$
\left|\alpha_{N} J_{F}\right\rangle \mapsto\left|j_{N} J_{F}\right\rangle \equiv\left|\left(J_{C}, j_{N}^{-1}\right) J_{F}\right\rangle,
$$

and

$$
E_{\alpha_{N} J_{F}} \mapsto E_{j_{N}} \equiv E_{C}-\epsilon_{j_{N}},
$$

where $\epsilon_{j_{N}}$ is the single-particle energy of state $j_{N}$. As an illustration, in the case of ${ }_{\Lambda}^{28} \mathrm{Si}$ the model space contains four single-particle states, both for protons and for neutrons $\left(n_{p}=n_{n}=4\right)$, namely, $1 s_{1 / 2}, 1 p_{3 / 2}, 1 p_{1 / 2}$, and $1 d_{5 / 2}$. Thus, for $\left|J_{C}\right\rangle=\left|1 d_{5 / 2} n^{-1}\right\rangle$, the final states (14) are constructed by 
adding two holes in the ${ }^{28} \mathrm{Si}$ nucleus and read:

$\begin{array}{ll}{ }_{\Lambda}^{28} \mathrm{Si} \rightarrow n n+{ }^{26} \mathrm{Si} & \stackrel{{ }_{\Lambda}^{28} \mathrm{Si} \rightarrow n p+{ }^{26} \mathrm{Al}}{ } \\ \left|\left(1 d_{5 / 2} n^{-1}\right)^{2} ; 0,2,4\right\rangle & \left|\left(1 d_{5 / 2} n^{-1} 1 d_{5 / 2} p^{-1}\right) ; 0,1,2,3,4,5\right\rangle \\ \left|1 d_{5 / 2} n^{-1} 1 s_{1 / 2} n^{-1} ; 2,3\right\rangle & \left|1 d_{5 / 2} n^{-1} 1 s_{1 / 2} p^{-1} ; 2,3\right\rangle \\ \left|1 d_{5 / 2} n^{-1} 1 p_{1 / 2} n^{-1} ; 2,3\right\rangle & \left|1 d_{5 / 2} n^{-1} 1 p_{1 / 2} p^{-1} ; 2,3\right\rangle \\ \left|1 d_{5 / 2} n^{-1} 1 p_{3 / 2} n^{-1} ; 1,2,3,4\right\rangle & \left|1 d_{5 / 2} n^{-1} 1 p_{3 / 2} p^{-1} ; 1,2,3,4\right\rangle .\end{array}$

The summation on $J_{F}$ in Eq. (9) can be performed for each single-particle state $j_{N}$, as done in Eqs. (11), (12), and (13) in Ref. [7]. One gets

$$
\begin{aligned}
& S_{n N}(E) \\
& \quad=\frac{4 M^{3}}{\pi} \sqrt{A(A-2)^{3}} \sum_{j_{N}} \sqrt{\left(\Delta_{j_{N}}-E\right)\left(E-\Delta_{j_{N}}^{\prime}\right)} \mathcal{F}_{j_{N}}(p P),
\end{aligned}
$$

where

$$
\mathcal{F}_{j_{N}}(p P)=\sum_{J=\left|j_{N}-1 / 2\right|}^{J=j_{N}+1 / 2} F_{j_{N}}^{J} \sum_{S l L \lambda T} \mathcal{M}^{2}\left(p P l L \lambda S J T ; j_{N}\right),
$$

with the spectroscopic factors $F_{j_{N}}^{J}$ exhibited in Table I of Ref. [7]. The maximum and minimum liberated energies are, respectively,

$$
\Delta_{\alpha_{N} J_{F}} \mapsto \Delta_{j_{N}}=\Delta+\epsilon_{j_{\Lambda}}+\epsilon_{j_{N}}
$$

and

$$
\Delta_{j_{N}}^{\prime}=\Delta_{j_{N}} \frac{A-2}{A},
$$

and the momenta are given by

$$
p=\sqrt{\frac{M A}{2}\left(E-\Delta_{j_{N}}^{\prime}\right)}
$$

and

$$
P=\sqrt{2 M(A-2)\left(\Delta_{j_{N}}-E\right)} .
$$

\section{B. IPSM-b}

Formally, one starts from the unperturbed basis $\left|i_{N} J_{F}\right\rangle_{0}$ with $i_{N}=1,2, \ldots n_{N}, n_{N}+1, \ldots$, where for $i_{N} \leqslant n_{N}$ we have the same simple doorway states $\left|j_{N} J_{F}\right\rangle$ in Eq. (14) (listed in Eq. (15) for ${ }_{\Lambda}^{28} \mathrm{Si}$ ), whereas for $i_{N} \geqslant n_{N}+1$ we have more complicated bound configurations (such as $3 \mathrm{~h} 1 \mathrm{p}$, $4 \mathrm{~h} 2 \mathrm{p}, \ldots$ in the case of ${ }_{\Lambda}^{28} \mathrm{Si}$ ) as well as those including unbound single-particle states in the continuum. As in Ref. [35], the perturbed eigenkets $\left|\alpha_{N} J_{F}\right\rangle$ and eigenvalues $E_{\alpha_{N} J_{F}}$ are obtained by diagonalizing the matrix ${ }_{0}\left\langle i_{N} J_{F}|H| i_{N}^{\prime} J_{F}\right\rangle_{0}$ of the exact Hamiltonian $H$ :

$$
\left\langle\alpha_{N} J_{F}|H| \alpha_{N}^{\prime} J_{F}\right\rangle=E_{\alpha_{N} J_{F}} \delta_{\alpha_{N} \alpha_{N}^{\prime}}
$$

with

$$
\begin{aligned}
\left|\alpha_{N} J_{F}\right\rangle & =\sum_{i_{N}=1}^{\infty} C_{i_{N}}^{\alpha_{N} J_{F}}\left|i_{N} J_{F}\right\rangle_{0} \\
& =\sum_{j_{N}} C_{j_{N}}^{\alpha_{N} J_{F}}\left|j_{N} J_{F}\right\rangle+\sum_{i_{N}=n_{N}+1}^{\infty} C_{i_{N}}^{\alpha_{N} J_{F}}\left|i_{N} J_{F}\right\rangle_{0} .
\end{aligned}
$$

It is easy to see that only the ket $\left|j_{N} J_{F}\right\rangle$ in the expansion (22) will contribute to the matrix element $\left\langle J_{C} \| a_{j_{N}}^{\dagger}|| \alpha_{N} J_{F}\right\rangle$ in Eq. (13). Therefore Eq. (9) takes the form

$$
\begin{aligned}
S_{n N}(E)= & \frac{4 M^{3}}{\pi} \sqrt{A(A-2)^{3}} \\
& \times \sum_{j_{N} \alpha_{N} J_{F}}\left|C_{j_{N}}^{\alpha_{N} J_{F}}\right|^{2} \sqrt{\left(\Delta_{\alpha_{N} J_{F}}-E\right)\left(E-\Delta_{\alpha_{N} J_{F}}^{\prime}\right)} \\
& \times \mathcal{F}\left(p, P ; j_{N} J_{F}\right),
\end{aligned}
$$

where

$$
\begin{aligned}
\mathcal{F}\left(p, P ; j_{N} J_{F}\right)= & \hat{J}_{I}^{-2} \sum_{l L \lambda S J T} \mid \mathcal{M}\left(p P l L \lambda S J T ; j_{N}\right) \\
& \times\left.\left\langle J_{I} \|\left(a_{j_{N}}^{\dagger} a_{j_{\Lambda}}^{\dagger}\right)_{J} \mid j_{N} J_{F}\right\rangle\right|^{2} .
\end{aligned}
$$

To evaluate the amplitudes $C_{i_{N}}^{\alpha_{N} J_{F}}$ one would have to choose the appropriate Hamiltonian $H$ and the unperturbed basis $\left|i_{N} J_{F}\right\rangle_{0}$ and solve the eigenvalue problem (21). We will not do this here. Instead, we will make a phenomenological estimate. First, because of the high density of states, we will convert the discrete energies $\Delta_{\alpha_{N} J_{F}}$ into the continuous variable $\varepsilon$, and the discrete sum on $\alpha_{N}$ into an integral on $\varepsilon$, i.e.,

$$
\begin{aligned}
\Delta_{\alpha_{N} J_{F}} & \rightarrow \varepsilon \\
\sum_{\alpha_{N} J_{F}}\left|C_{j_{N}}^{\alpha_{N} J_{F}}\right|^{2} & \rightarrow \sum_{J_{F}} \int_{-\infty}^{\infty}\left|C_{j_{N} J_{F}}(\varepsilon)\right|^{2} \rho_{J_{F}}(\varepsilon) d \varepsilon
\end{aligned}
$$

where $\rho_{J_{F}}(\varepsilon)$ is the density of perturbed states with angular momentum $J_{F}$. In this way the spectrum (23) becomes

$$
\begin{aligned}
S_{n N}(E)= & \frac{4 M^{3}}{\pi} \sqrt{A(A-2)^{3}} \\
& \times \sum_{j_{N} J_{F}} \int_{-\infty}^{\infty} \mathrm{P}_{j_{N} J_{F}}(\varepsilon) \sqrt{(\varepsilon-E)\left(E-\varepsilon^{\prime}\right)} \\
& \times \mathcal{F}\left(p, P ; j_{N} J_{F}\right) d \varepsilon,
\end{aligned}
$$


where

$$
P_{j_{N} J_{F}}(\varepsilon)=\left|C_{j_{N} J_{F}}(\varepsilon)\right|^{2} \rho_{J_{F}}(\varepsilon)
$$

is called the strength function [35-37] and represents the probability of finding the configuration $\left|j_{N} J_{F}\right\rangle \equiv\left|\left(J_{C}, j_{N}^{-1}\right) J_{F}\right\rangle$ per unit energy interval. Moreover,

$$
\begin{aligned}
p & =\sqrt{\frac{M A}{2}\left(E-\varepsilon^{\prime}\right),} \\
P & =\sqrt{2 M(A-2)(\varepsilon-E),} \\
\varepsilon^{\prime} & =\varepsilon \frac{A-2}{A},
\end{aligned}
$$

and the condition

$$
\varepsilon^{\prime} \leqslant E \leqslant \varepsilon
$$

has to be fulfilled throughout the $\varepsilon$ integration. It is convenient to introduce the averaged strength function

$$
P_{j_{N}}(\varepsilon)=\frac{1}{\operatorname{dim}\left(j_{N} J_{C}\right)} \sum_{J_{F}=\left|J_{C}-j_{N}\right|}^{J_{C}+j_{N}} P_{j_{N} J_{F}}(\varepsilon)
$$

where

$$
\operatorname{dim}\left(j_{N} J_{C}\right)= \begin{cases}2 j_{N}+1 & \text { for } j_{N} \leqslant J_{C} \\ 2 J_{C}+1 & \text { for } J_{C}<j_{N}\end{cases}
$$

This allows to simplify Eq. (26) by making the approximation $P_{j_{N} J_{F}}(\varepsilon) \approx P_{j_{N}}(\varepsilon)$ to get

$$
S_{n N}(E)=\frac{4 M^{3}}{\pi} \sqrt{A(A-2)^{3}}
$$

$$
\times \sum_{j_{N}} \int_{-\infty}^{\infty} P_{j_{N}}(\varepsilon) \sqrt{(\varepsilon-E)\left(E-\varepsilon^{\prime}\right)} \mathcal{F}_{j_{N}}(p P) d \varepsilon
$$

where we noticed that the summation of $\mathcal{F}\left(p, P ; j_{N} J_{F}\right)$ over $J_{F}$ gives the function defined in Eq. (17).

The IPSM-a results would be recovered if one made the further approximation

$$
P_{j_{N}}(\varepsilon)=\delta\left(\varepsilon-\Delta_{j_{N}}\right) .
$$

Here, in IPSM-b, the $\delta$ functions (34) will be used for the strictly stationary states, whereas for the fragmented hole states we will use Breit-Wigner distributions,

$$
P_{j_{N}}(\varepsilon)=\frac{2 \gamma_{j_{N}}}{\pi} \frac{1}{\gamma_{j_{N}}^{2}+4\left(\varepsilon-\Delta_{j_{N}}\right)^{2}}, \int_{-\infty}^{\infty} P_{j_{N}}(\varepsilon) d \varepsilon=1
$$

where $\gamma_{j_{N}}$ are the widths of the resonance centroids at energies $\Delta_{j_{N}}$ (see Eq.(2.11.22) in Ref. [35]).

It might be important to point out that, because both strength functions $P_{j_{N}}(\varepsilon)$ are normalized to unit, their effect on integrated observables like the decay rates $\Gamma_{N}$ is expected to be small even if they considerably affect the spectra. This will be further investigated in Sec. IV.

\section{NUMERICAL RESULTS}

In Figs. 1 and 2 we show, respectively, the normalized energy spectra $S_{n p}(E) / \Gamma_{p}$ and $S_{n n}(E) / \Gamma_{n}$ for ${ }_{\Lambda}^{4} \mathrm{He}$, ${ }_{\Lambda}^{5} \mathrm{He},{ }_{\Lambda}^{12} \mathrm{C},{ }_{\Lambda}^{16} \mathrm{O}$, and ${ }_{\Lambda}^{28} \mathrm{Si}$ hypernuclei, evaluated within the full OMEP, that comprises the $\left(\pi, \eta, K, \rho, \omega, K^{*}\right)$ mesons.

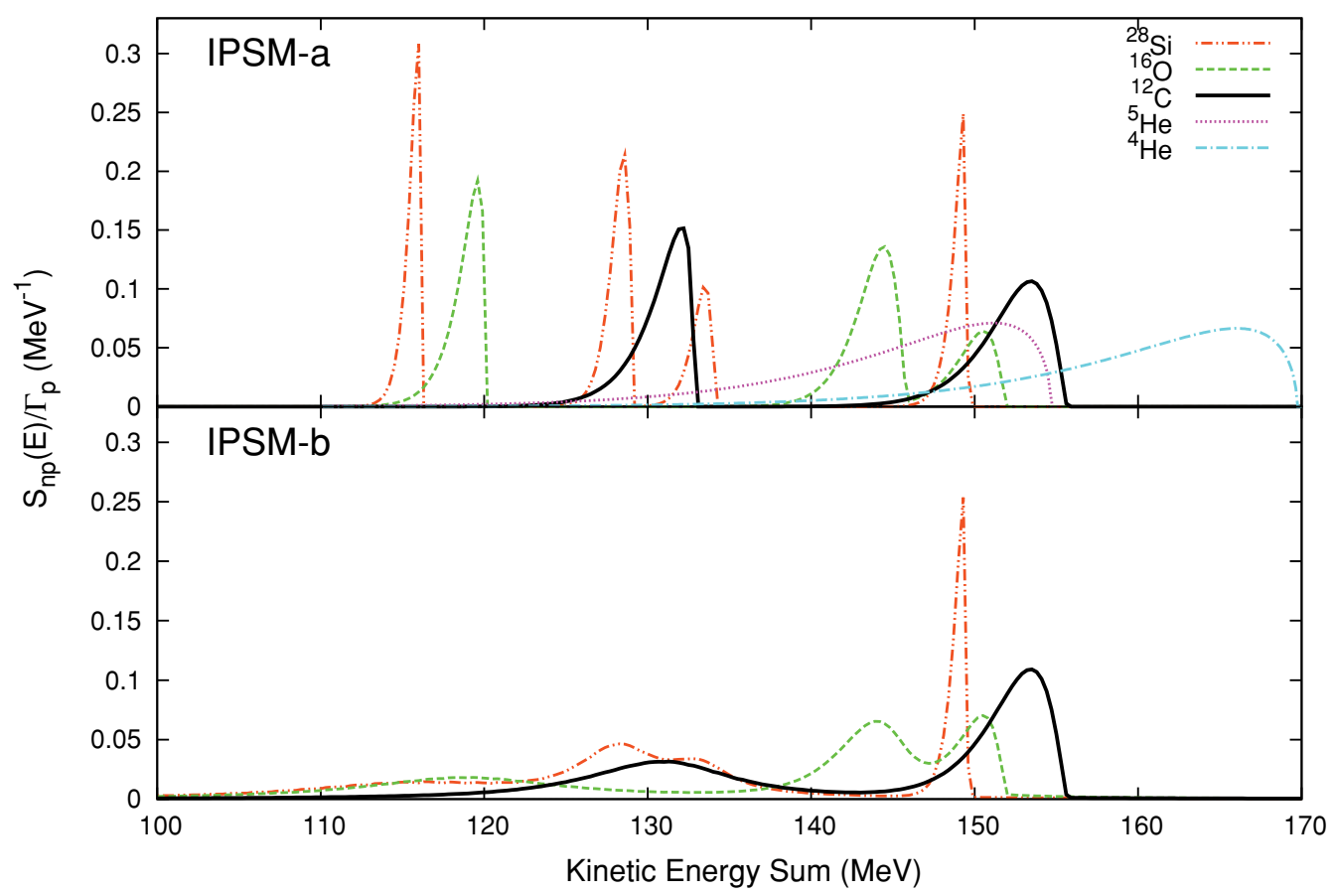

FIG. 1. (Color online) Normalized energy spectra $S_{n p}(E) / \Gamma_{p}$ for ${ }_{\Lambda}^{4} \mathrm{He},{ }_{\Lambda}^{5} \mathrm{He},{ }_{\Lambda}^{12} \mathrm{C},{ }_{\Lambda}^{16} \mathrm{O}$, and ${ }_{\Lambda}^{28} \mathrm{Si}$ hypernuclei for the full OMEP obtained within the approaches IPSM-a (upper panel) and IPSM-b (lower panel). For the $s$-shell hypernuclei, only the IPSM-a approach has been used. 


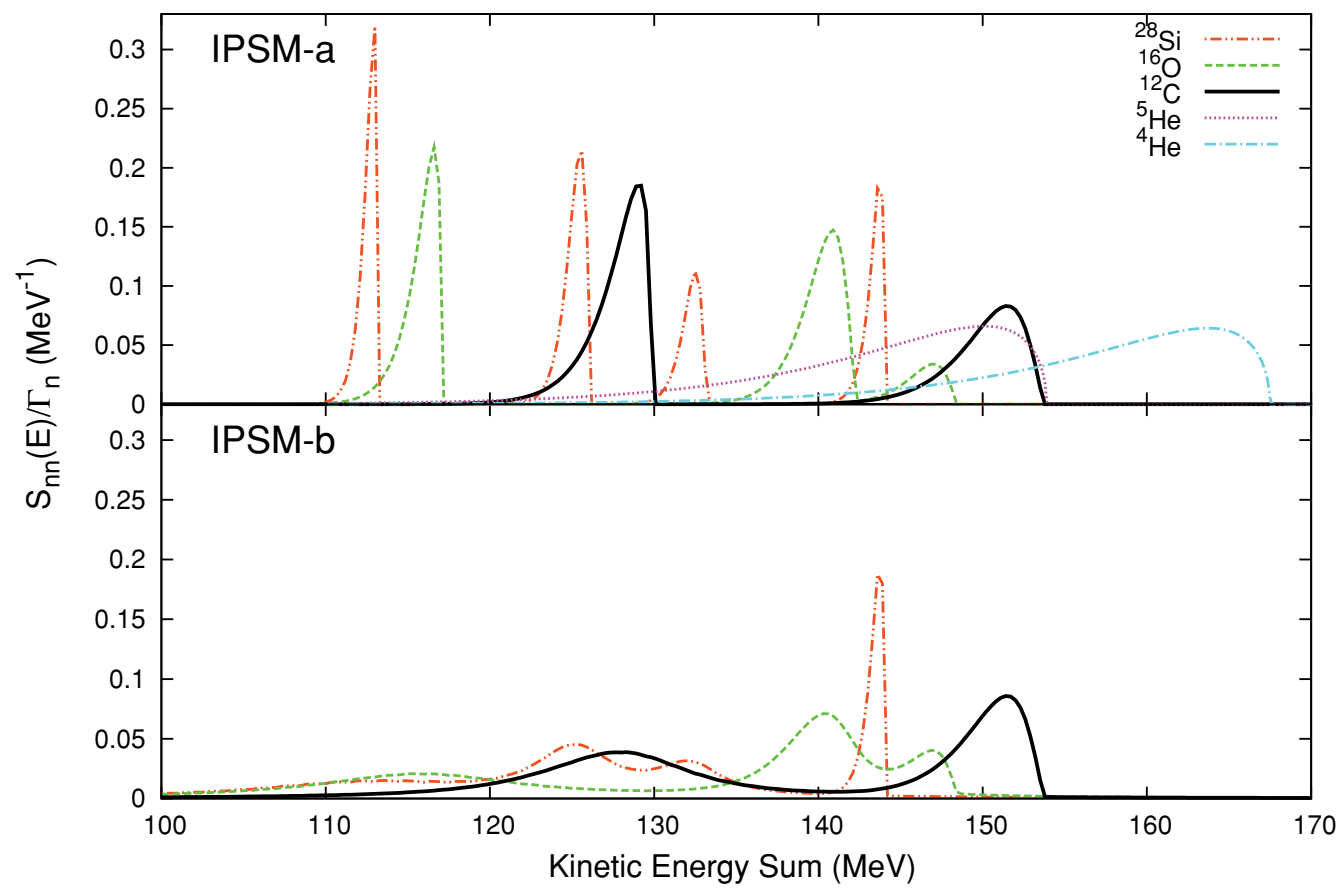

FIG. 2. (Color online) Normalized energy spectra $S_{n n}(E) / \Gamma_{n}$ for ${ }_{\Lambda}^{4} \mathrm{He},{ }_{\Lambda}^{5} \mathrm{He},{ }_{\Lambda}^{12} \mathrm{C},{ }_{\Lambda}^{16} \mathrm{O}$, and ${ }_{\Lambda}^{28}$ Si hypernuclei for the full OMEP, obtained within the approaches IPSM-a (upper panel) and IPSM-b (lower panel). For the $s$-shell hypernuclei, only the IPSM-a approach has been used.

The single-particle energies for the strictly stationary hole states have been taken from Wapstra and Gove's compilation [38], and those of the quasi-stationary ones have been estimated from the studies of the quasi-free scattering processes $(p, 2 p)$ and $\left(e, e^{\prime} p\right)$ [26-34].

The two IPSM approaches exhibit some quite important differences:

(i) IPSM- $a$ : The spectra cover the energy region $110 \mathrm{MeV}$ $<E<170 \mathrm{MeV}$ and contain one or more peaks, the number of which is equal to the number of shell-model orbitals $1 s_{1 / 2}, 1 p_{3 / 2}, 1 p_{1 / 2}, 1 d_{5 / 2}, 2 s_{1 / 2}, 1 d_{3 / 2} \cdots$ that are either fully or partly occupied in $\left|J_{C}\right\rangle$. Before including the recoil, all these peaks would be just spikes at the liberated energies $\Delta_{j_{N}}$, as can be seen from Eq. (3) setting $E_{r}=0$. With the recoil effect, they behave as

$$
\begin{aligned}
S_{n N}\left(E \cong \Delta_{j_{N}}\right) \sim & \sqrt{\left(\Delta_{j_{N}}-E\right)\left(E-\Delta_{j_{N}}^{\prime}\right)} \\
& \times e^{-M(A-2)\left(\Delta_{j_{N}}-E\right) b^{2}}
\end{aligned}
$$

and develop rather narrow widths $\sim\left[b^{2} M(A-2)\right]^{-1}$, where $b$ is the harmonic oscillator size parameter, which has been taken from Ref. [5]. These widths go from $\cong 3 \mathrm{MeV}$ for ${ }_{\Lambda}^{28} \mathrm{Si}$ to $\cong 20 \mathrm{MeV}$ for ${ }_{\Lambda}^{4} \mathrm{He}$, as indicated in the upper panels of the just mentioned figures.

(ii) IPSM- $b$ : In the lower panels of the same figures are shown the results obtained when the recoil is convoluted with the Breit-Wigner distributions (35) for the strength functions of the fragmented deep hole states. The widths $\gamma_{j_{N}}$ have been estimated from Refs. [26-35], and in particular from Figure 11 of Ref. [26] and
Table 1 of [30], with the results: $\gamma_{1 s_{1 / 2}}=9 \mathrm{MeV}$ in ${ }_{\Lambda}^{12} \mathrm{C}, \gamma_{1 s_{1 / 2}}=14 \mathrm{MeV}$ and $\gamma_{1 p_{3 / 2}}=3 \mathrm{MeV}$ in ${ }_{\Lambda}^{16} \mathrm{O}^{4}$ and $\gamma_{1 s_{1 / 2}}=16 \mathrm{MeV}$ and $\gamma_{1 p_{3 / 2}}=\gamma_{1 p_{1 / 2}}=5 \mathrm{MeV}$ in ${ }_{\Lambda}^{28} \mathrm{Si}$, both for protons and neutrons. One sees that, except for the ground states, the narrow peaks engendered by the recoil effect become now pretty wide bumps.

We feel that the above rather rudimentary parametrization could be realistic enough for a qualitative discussion of the kinetic energy sum spectra. A more accurate model should be probably necessary for a full quantitative study and comparison with data.

\section{GENERAL CONSIDERATIONS AND CONNECTION WITH DATA}

The normalized spectra shown in Figs. 1 and 2 have a very weak dependence on the dynamics involved in the NMWD process proper, and almost identical shapes would have been obtained if only the one-pion exchange potential (OPEP) had been taken into account. To understand this fact one can appeal to the s-wave approximation, which assumes that only the relative matrix elements of the form $\langle p, l S J T|V| 0 J J T\rangle$, i.e., with the $\Lambda N$ system in an s-state, significantly contribute to $\mathcal{M}\left(p P l L \lambda S J T ; j_{N}\right)$. This has been examined quantitatively, for $p$-shell hypernuclei, in Ref. [39]; see also the Refs. [5,6]. Furthermore, as we have discussed in Ref. [25], those matrix elements depend only very weakly on the relative momentum

\footnotetext{
${ }^{4}$ The $3 / 2_{1}^{-}$peak is at $6.32 \mathrm{MeV}$, but small amounts of the $p_{3 / 2}$ strength are also fragmented to the states of 9.93 and $10.7 \mathrm{MeV}$ [34].
} 


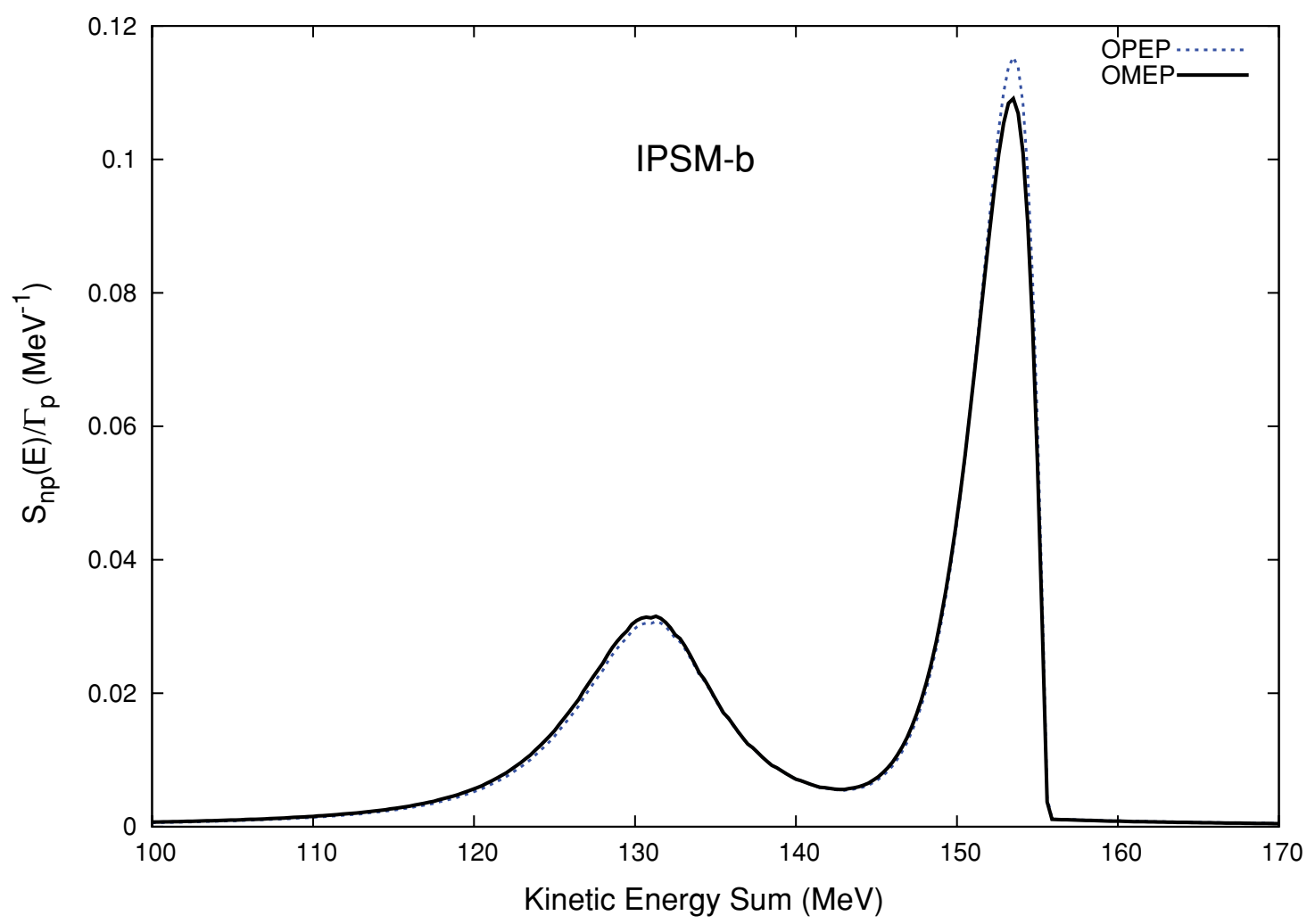

FIG. 3. (Color online) Normalized energy spectra $S_{n p}(E) / \Gamma_{p}$ for the decay of ${ }_{\Lambda}^{12} \mathrm{C}$, computed with the full OMEP and the OPEP in the framework of the IPSM-b.

$p$ and, as such, they can be evaluated at the maximum value of $p=p_{\Delta}=\sqrt{M \Delta}$, which corresponds to $P=0$ or, according to Eq. (20), to $E=\Delta_{j_{N}} \cong \Delta$. Thus the energy dependence of $S_{n N}(E) / \Gamma_{N}$ remains exclusively in kinematical phase-space factors, and the position and width of the peaks will be unaffected by the dynamics of the decay process, which will influence, to some extent, only their relative heights. This is illustrated in the case of the $n p$ spectrum for ${ }_{\Lambda}^{12} \mathrm{C}$ in Fig. 3, showing that even this latter effect is very small. The comparison between the normalized spectra obtained with the full OMEP and with the $\pi+K$ exchange potential would give an almost perfect superposition.

The transition probabilities $\Gamma_{N}$ strongly depend on the hypernuclear transition potential, but this dependence is washed out in the ratios defining the normalized spectra. Conversely, for a given choice of transition potential both shell-model approaches discussed here yield very similar results for the $\Gamma_{N}$. These points are illustrated in Table I, where one can also see that, as anticipated in Sec. II, the effect of the recoil on these quantities is negligible.

One can then summarize our findings by saying that in light systems $\left({ }_{\Lambda}^{4} \mathrm{He}\right.$ and $\left.{ }_{\Lambda}^{5} \mathrm{He}\right)$ the kinetic energy sum coincidence spectra $S_{n N}(E)$, normalized to the total decay rates $\Gamma_{N}$, basically depend on energies associated with the three-body kinematics. The differences between $S_{n p}(E)$ and $S_{n n}(E)$ are mainly due to the differences in the proton and neutron separation energies and in the spectroscopic factors $F_{j_{N}}^{J}$. For the remaining hypernuclei it is imperative, in addition,
TABLE I. Nonmesonic decay rates in units of $\Gamma_{\Lambda}^{0}=2.50 \times$ $10^{-6} \mathrm{eV}$ and $n / p$ branching ratios for ${ }_{\Lambda}^{12} \mathrm{C},{ }_{\Lambda}^{16} \mathrm{O}$, and ${ }_{\Lambda}^{28} \mathrm{Si}$ computed with several transition potentials and using the IPSM-a and IPSM-b approaches. The values obtained in the IPSM-a framework but neglecting the recoil are shown within parentheses.

\begin{tabular}{|c|c|c|c|}
\hline & $\Gamma_{n}$ & $\Gamma_{p}$ & $\Gamma_{n} / \Gamma_{p}$ \\
\hline \multicolumn{4}{|c|}{${ }_{\Lambda}^{12} \mathrm{C}$ IPSM-a } \\
\hline OMEP & $0.249(0.249)$ & $0.956(0.960)$ & $0.260(0.259)$ \\
\hline$\pi+K$ & $0.244(0.244)$ & $0.755(0.758)$ & $0.323(0.322)$ \\
\hline \multicolumn{4}{|c|}{${ }_{\Lambda}^{12} \mathrm{C}$ IPSM-b } \\
\hline OMEP & 0.246 & 0.947 & 0.260 \\
\hline$\pi+K$ & 0.241 & 0.748 & 0.322 \\
\hline OPEP & 0.142 & 1.004 & 0.141 \\
\hline \multicolumn{4}{|c|}{${ }_{\Lambda}^{16} \mathrm{O}$ IPSM-a } \\
\hline OMEP & $0.290(0.290)$ & $1.024(1.027)$ & $0.283(0.282)$ \\
\hline$\pi+K$ & $0.287(0.287)$ & $0.811(0.813)$ & $0.354(0.353)$ \\
\hline \multicolumn{4}{|c|}{${ }_{\Lambda}^{16} \mathrm{O}$ IPSM-b } \\
\hline OMEP & 0.285 & 1.009 & 0.282 \\
\hline$\pi+K$ & 0.282 & 0.799 & 0.353 \\
\hline \multicolumn{4}{|c|}{${ }_{\Lambda}^{28} \mathrm{Si}$ IPSM-a } \\
\hline OMEP & $0.348(0.348)$ & $1.163(1.164)$ & $0.299(0.299)$ \\
\hline$\pi+K$ & $0.341(0.341)$ & $0.934(0.935)$ & $0.365(0.365)$ \\
\hline \multicolumn{4}{|c|}{${ }_{\Lambda}^{28} \mathrm{Si}$ IPSM-b } \\
\hline OMEP & 0.345 & 1.123 & 0.307 \\
\hline$\pi+K$ & 0.338 & 0.903 & 0.374 \\
\hline
\end{tabular}


to take into account that most of the hole states are fragmented and consequently one has to consider the spreading of their strengths.

The residual interaction among the valence particles and their coupling to the collective rotational and/or vibrational motions are not explicitly considered in the present work. But it is not expected that these would modify qualitatively the above scenario. It might be worth noting, nevertheless, that the pairing force would be capable of shifting some of the strength from the occupied levels to higher lying orbitals. For instance, in ${ }_{\Lambda}^{12} \mathrm{C}$ a part of the $1 p_{3 / 2}$ strength would be moved up into the $1 p_{1 / 2}$ orbital, whereas in ${ }_{\Lambda}^{28} \mathrm{Si}$ the strength would be moved from the $1 d_{5 / 2}$ level into the empty $2 s_{1 / 2}$ and $1 d_{3 / 2}$ states. However, we believe that the coupling of the deep-hole states to other more complicated configurations through the residual interaction, which is treated here in a phenomenological way, is by far a more relevant effect for the physics discussed in the present article.

We would not like to end this article without making some comments on the relation between the formalism developed here and the experiments. To this end we shall follow closely the discussion in Ref. [40]. The theoretical prediction for the number of $n N$ pairs detected in coincidence with kinetic energy sum $E$ within the interval $d E$ can be written as

$$
d N_{n N}(E)=C_{n N}(E) S_{n N}(E) d E,
$$

where the factor $C_{n N}(E)$ depends on the experimental environment and includes all quantities and effects not considered in $S_{n N}(E)$, such as the number of produced hypernuclei, the detection efficiency and acceptance, and so on. Assuming, for simplicity, that the experimental spectra have already been corrected for detection efficiency and acceptance and that the possible remaining energy dependence in this factor can be neglected, the predicted total number of detected events $N_{n N}$ can be related to $\Gamma_{N}$ as follows:

$$
N_{n N}=\int \frac{d N_{n N}(E)}{d E} d E=C_{n N} \int S_{n N}(E) d E=C_{n N} \Gamma_{N} .
$$

This allows us to rewrite (37) in the form

$$
\frac{d N_{n N}(E)}{d E}=N_{n N} \frac{S_{n N}(E)}{\Gamma_{N}} \text {. }
$$

What is measured in an experiment is the number of pairs $\Delta N_{n N}^{\exp }\left(E_{i}\right)$ at a given energy $E_{i}$ within a fixed energy bin $\Delta E_{n N}$, i.e., $\Delta N_{n N}^{\exp }\left(E_{i}\right) / \Delta E_{n N}$. The total number of observed events is

$$
N_{n N}^{\exp }=\sum_{i=1}^{m} \Delta N_{n N}^{\exp }\left(E_{i}\right)
$$

where $m$ is the number of bins. The spectrum $S_{n N}(E)$ can be normalized to the experimental one by identifying $N_{n N}$ in
Eq. (39) with $N_{n N}^{\exp }$. Thus, the quantity that we have to confront with measurements is

$$
\Delta N_{n N}(E)=N_{n N}^{\exp } \Delta E_{n N} \frac{S_{N}(E)}{\Gamma_{N}} .
$$

For instance, to compare the experimental data given in Figure 11 in Ref. [23] with our calculations shown in Figs. 1 and 2, the latter should be multiplied by the factors $N_{n p}^{\text {exp }}$. $\Delta E_{n p}=87 \times 5 \mathrm{MeV}=435 \mathrm{MeV}$, and $N_{n n}^{\exp } \cdot \Delta E_{n n}=19 \times$ $5 \mathrm{MeV}=95 \mathrm{MeV}$, respectively.

Here we have, neither considered the resolution of the detector system, nor explicitly included the FSI. Also, we have ignored the three-body $\Lambda N N$ decay contributions. As a consequence it is very reasonable that only a wide bump at about $140 \mathrm{MeV}$ would appear in the experimental spectra for ${ }_{\Lambda}^{12} \mathrm{C}$ and heavier hypernuclei. The IPSM predicts quite similar spectra for the $n p$ and $n n$ pairs. Moreover, from the present results one could venture to say that the neutron bump should lie at a smaller energy than the proton one. This agrees only marginally with the experiments performed so far, where important differences between the $n p$ and $n n$ spectra have been observed.

\section{CONCLUDING REMARKS}

In this article, we investigated, in the framework of the IPSM, the effects of the recoil of the residual nucleus and of the spreading in strength of the deep-hole states on NMWD observables. We conclude that, although their effect is of minor importance for integrated observables like the decay rates and the $n / p$ branching ratio, they play a crucial role in determining the shapes of the normalized kinetic energy sum coincidence spectra of $n n$ and $n p$ pairs. For the spectra of $s$-shell hypernuclei, the recoil effect is the most important one.

In summary, we believe that the IPSM is the appropriate lowest-order approximation for the theoretical calculation of the two-particle spectra in the NMWD when (a) the recoil effect is included and (b) the fragmentation of the strengths of the deep-hole states is taken into account. It is in comparison to this picture that one should appraise the effects of the FSI and of the two-nucleon-induced decay mode.

The consequences of the two effects dealt with here on the one-particle kinetic energy spectra and on the opening angle distributions of $n p$ and $n n$ pairs will be discussed elsewhere.

\section{ACKNOWLEDGMENTS}

This work was partly supported by the Brazilian agencies CNPq and FAPESP and by the Argentinian agency CONICET under contract PIP 6159. M. S. Hussein thanks the Martin Gutzwiller program at the Max Plank Institute for the Physics of Complex Systems-Dresden for support.
[1] J. J. Szymanski, P. D. Barnes, G. E. Diebold, R. A. Eisenstein, G. B. Franklin, R. Grace, D. W. Hertzog, C. J. Maher, B. P. Quinn, R. Rieder, J. Seydoux, W. R. Wharton, S. Bart, R. E. Chrien, P. Pile, R. Sutter, Y. Xu, R. Hackenburg, E. V. Hungerford,
T. Kishimoto, L. G. Tang, B. Bassalleck, and R. L. Stearns, Phys. Rev. C 43, 849 (1991).

[2] H. Noumi, S. Ajimura, H. Ejiri, A. Higashi, T. Kishimoto, D. R. Gill, L. Lee, A. Olin, 
T. Fukuda, and O. Hashimoto, Phys. Rev. C 52, 2936 (1995).

[3] O. Hashimoto, S. Ajimura, K. Aoki, H. Bhang, T. Hasegawa, H. Hotchi, Y. D. Kim, T. Kishimoto, K. Maeda, H. Noumi, Y. Ohta, K. Omata, H. Outa, H. Park, Y. Sato, M. Sekimoto, T. Shibata, T. Takahashi, and M. Youn, Phys. Rev. Lett. 88, 042503 (2002).

[4] A. Parreño and A. Ramos, Phys. Rev. C 65, 015204 (2001).

[5] K. Itonaga, T. Ueda, and T. Motoba, Phys. Rev. C 65, 034617 (2002).

[6] C. Barbero, D. Horvat, F. Krmpotić, T. T. S. Kuo, Z. Narančić, and D. Tadić, Phys. Rev. C 66, 055209 (2002).

[7] F. Krmpotić and D. Tadić, Braz. J. Phys. 33, 187 (2003).

[8] C. Barbero, C. De Conti, A. P. Galeão, and F. Krmpotić, Nucl. Phys. A726, 267 (2003).

[9] E. Bauer and F. Krmpotić, Nucl. Phys. A717, 217 (2003); A739, 109 (2004).

[10] C. Barbero, A. P. Galeão, and F. Krmpotić, Phys. Rev. C 72, 035210 (2005).

[11] G. Garbarino, arXiv:nucl-th/0701049.

[12] J. H. Kim et al., Phys. Rev. C 68, 065201 (2003).

[13] S. Okada et al., Phys. Lett. B597, 249 (2004).

[14] G. Garbarino, A. Parreño, and A. Ramos, Phys. Rev. Lett. 91, 112501 (2003).

[15] G. Garbarino, A. Parreño, and A. Ramos, Phys. Rev. C 69, 054603 (2004).

[16] G. Garbarino, A. Parreño, and A. Ramos, Nucl. Phys. A754, 137c (2005).

[17] H. Outa et al., Nucl. Phys. A754, 157c (2005).

[18] S. Okada et al., Nucl. Phys. A752, 169c (2005).

[19] B. H. Kang et al., Phys. Rev. Lett. 96, 062301 (2006).

[20] M. J. Kim et al., Phys. Lett. B641, 28 (2006).

[21] E. Bauer, G. Garbarino, A. Parreño, and A. Ramos, arXiv:nuclth/0602066.

[22] E. Bauer, Nucl. Phys. A781, 424 (2007); A796, 11 (2007).
[23] J. D. Parker et al., Phys. Rev. C 76, 035501 (2007).

[24] H. Bhang et al., Eur. Phys. J. A 33, 259 (2007).

[25] C. Barbero, A. P. Galeão, and F. Krmpotić, Phys. Rev. C 76, 054321 (2007).

[26] G. Jacob and T. A. J. Maris, Rev. Mod. Phys. 45, 6 (1973).

[27] S. Frullani and J. Mougey, Adv. Nucl. Phys. 14, 1 (1984).

[28] S. L. Belostotskii et al., Sov. J. Nucl. Phys. 41, 903 (1985); S. S. Volkov et al., Sov. J. Nucl. Phys. 52, 848 (1990).

[29] M. Leuschner et al., Phys. Rev. C 49, 955 (1994).

[30] T. Yamada, M. Takahashi, and K. Ikeda, Phys. Rev. C 53, 752 (1996).

[31] T. Yamada, Nucl. Phys. A687, 297c (2001).

[32] M. Yosoi, H. Akimune, I. Daito, H. Ejiri, H. Fujimura, M. Fujiwara, T. Ishikawa, M. Itoh, T. Kawabata, M. Nakamura, T. Noro, E. Obayashi, H. Sakaguchi, H. Takeda, T. Taki, A. Tamii, H. Toyokawa, N. Tsukahara, M. Uchida, T. Yamada, and H. P. Yoshida, Phys. Lett. B551, 255 (2003).

[33] T. Yamada, M. Yosoi, and H. Toyokawa, Nucl. Phys. A738, 323 (2004).

[34] K. Kobayashi, H. Akimune, H. Ejiri, H. Fujimura, M. Fujiwara, K. Hara, K. Y. Hara, T. Ishikawa, M. Itoh, Y. Itow, T. Kawabata, M. Nakamura, H. Sakaguchi, Y. Sakemi, M. Shiozawa, H. Takeda, Y. Totsuka, H. Toyokawa, M. Uchida, T. Yamada, Y. Yasuda, H.P. Yoshida, M. Yosoi, and R. G. T. Zegers, arXiv:nucl-ex/0604006.

[35] C. Mahaux, P. E. Bortignon, R. A. Broglia, and C. H. Dasso, Phys. Rep. 120, 1 (1985).

[36] N. Frazier, B. A. Brown, and V. Zelevinsky, Phys. Rev. C 54, 1665 (1996).

[37] A. J. Sargeant, M. S. Hussein, M. P. Pato, and M. Ueda, Phys. Rev. C 61, 011302(R) (1999).

[38] A. H. Wapstra and N. B. Gove, Nucl. Data Tables 9, 265 (1971).

[39] C. Bennhold and A. Ramos, Phys. Rev. C 45, 3017 (1992).

[40] E. Bauer, A. P. Galeão, M. S. Hussein, and F. Krmpotić, arXiv:0808.0531. 